\title{
Áreas restauradas revelam nova ocorrência de Neofavolus subpurpurascens (Murrill) Palacio \& Robledo (Basidiomycota, Polyporaceae) para o Estado de São Paulo, Brasil
}

\author{
- $\underline{\text { Alex Almeida Alcantara }}^{1,2},{ }^{- \text {Adriana de Mello Gugliotta }}{ }^{1}$ e Luiz Mauro Barbosa ${ }^{1}$
}

Recebido: 25 junho 2019; aceito: 23 outubro 2019

Como citar: Alcantara, A.A., Gugliotta, A.M. \& Barbosa, L.M. 2019. Áreas restauradas revelam nova ocorrência de Neofavolus subpurpurascens (Murrill) Palacio \& Robledo (Basidiomycota, Polyporaceae) para o Estado de São Paulo, Brasil. Hoehnea 46: e732019. http://dx.doi.org/10.1590/2236-8906-73/2019.

ABSTRACT - (Restored areas reveal a new record from São Paulo State, Brazil: Neofavolus subpurpurascens (Murrill) Palacio \& Robledo (Basidiomycota, Polyporaceae)). The diversity of wood-inhabiting Agaricomycetes (Basidiomycota) was studied in the Parque Florestal São Marcelo, Brazilian area under restoration since 2002 by the planting of more than 100 native tree species, located in the municipality of Mogi-Guaçu (São Paulo State). Collections were made bi-monthly bimonthly from October 2015 to October 2016. The identification of the samples was based on the morphological analysis of the basidiomata; the selected specimens were deposited at the SP Herbarium. During the study, a total of 224 specimens was identified as belonging to 41 distinct species, including one new record for the State of São Paulo: Neofavolus subpurpurascens (Murrill) Palacio \& Robledo. The results, show the establishment of the fungal community in the restored area and corroborate the contribution of that the reforestation of degraded areas to the maintenance of the fungal diversity of the state. Keywords: Basidiomycota, conservation, diversity, Polyporaceae, reforestation

RESUMO - (Áreas restauradas revelam nova ocorrência de Neofavolus subpurpurascens (Murrill) Palacio \& Robledo (Basidiomycota, Polyporaceae) para o Estado de São Paulo, Brasil). A diversidade de Agaricomycetes lignícolas (Basidiomycota) foi estudada no Parque Florestal São Marcelo, localizado no município de Mogi-Guaçu, SP, Brasil, em processo de restauração desde 2002 por meio de um plantio com mais de 100 espécies arbóreas nativas. As coletas foram realizadas bimestralmente durante o período de outubro de 2015 a outubro de 2016. Os espécimes foram identificados com base na morfologia do basidioma e depositados no Herbário SP. Durante o estudo, 224 espécimes foram identificados em 41 espécies, dentre as quais um novo registro para o Estado de São Paulo: Neofavolus subpurpurascens (Murrill) Palacio \& Robledo, mostrando o estabelecimento da comunidade fúngica na área restaurada e ainda que a recuperação de áreas degradadas contribui para a manutenção da diversidade fúngica do Estado.

Palavras-chave: Basidiomycota, conservação, diversidade, Polyporaceae, reflorestamento

\section{Introdução}

O conhecimento da diversidade de macrofungos, no Estado de São Paulo, especialmente de Agaricomycetes, tem se restringido às unidades de conservação (Bononi 1984, Gugliotta \& Bononi 1999, Leal \& Gugliotta 2008, Gugliotta et al. 2011, Abrahão et al. 2012, Motato-Vásquez \& Gugliotta 2013, Motato-Vásquez et al. 2014, Pires \& Gugliotta 2016). A Reserva Particular do Patrimônio Natural (RPPN) Parque Florestal São Marcelo localizada no município de Mogi-Guaçu, São Paulo, foi implementada em 2002, quando uma área de 240 ha, pertencente à empresa International Paper, foi destinada à restauração (Barbosa et al. 2013).

Anteriormente à aquisição pela empresa em 1995, a propriedade de 822 ha era destinada ao plantio de culturas de ciclo anual, semi-perenes, como cana-deaçúcar, e perenes, como café, citrus, além de pastagem. Após a aquisição, a área foi destinada unicamente ao plantio de Eucalyptus sp., até o período de 2002, quando a área de 240 ha foi destinada à implantação da

1. Instituto de Botânica, Caixa Postal 68041, 04045-972 São Paulo, SP, Brasil

2. Autor para correspondência: alex.ex100@gmail.com 
RPPN. As áreas circunvizinhas à RPPN são compostas por propriedades rurais, incluindo o Horto MogiGuaçu pertencente à empresa, destinado ao plantio comercial de Eucalyptus sp., existindo pequenos fragmentos florestais representados por faixas ciliares de vegetação nativa remanescente, o que torna a RPPN importante para o estabelecimento da conectividade da paisagem.

De acordo com Barbosa et al. (2013), foi implantada na reserva um reflorestamento heterogêneo de alta diversidade com 101 espécies arbóreas nativas (56\% não pioneiras, $37 \%$ pioneiras e $7 \%$ de espécies não classificadas ou exóticas), escolhidas dentre as que ocorrem na fitofisionomia Floresta Estacional Semidecidual, que anteriormente cobria a região (Mandetta 2007).

O presente estudo está inserido em um projeto amplo desenvolvido pela Coordenação Especial para Restauração de Áreas Degradadas (CERAD) do Instituto de Botânica, que visa o monitoramento da restauração da RPPN por meio de pesquisas em parcelas permanentes.

Após nove anos de instalação, os estudos de monitoramento utilizando parâmetros como a área basal, altura média e diversidade vegetal, revelaram que a RPPN apresenta um estrato arbóreo bem desenvolvido. No entanto, por ser uma floresta recente, seu estrato arbóreo está majoritariamente caracterizado pela composição de espécies utilizadas no plantio (Mandetta 2007, Colmanetti \& Barbosa 2013, Colmanetti et al. 2016).

Para verificar se o reflorestamento foi eficiente e se a área está recuperando as suas funções ecológicas, estudos de monitoramento na área são realizados, procedimento essencial na certificação da eficiência das ações restauradoras. No entanto, a grande maioria dos parâmetros utilizados como indicadores refere-se à comunidade vegetal (Colmanetti \& Barbosa 2013, Gandolfi 2013) ou à presença de fauna, normalmente associada à dispersão das espécies vegetais (Silveira et al. 2011). Apesar da grande importância ecológica dos fungos lignícolas na ciclagem de nutrientes e na manutenção dos ecossistemas terrestres (Rayner 1995, Boddy et al. 2008), estes organismos não têm sido utilizados como parâmetro de monitoramento da restauração florestal.

O objetivo deste trabalho é ampliar o conhecimento e a distribuição geográfica de Neofavolus subpurpurascens (Murrill) Palacio \& Robledo, até então não registrada para o Estado de
São Paulo e cuja ocorrência foi verificada em uma área em restauração no Estado (Alcantara 2017).

\section{Material e métodos}

Área estudada - A Reserva Particular de Patrimônio Natural (RPPN) Parque Florestal São Marcelo (figura 1), localiza-se no município de Mogi-Guaçu,

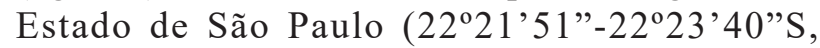
$\left.46^{\circ} 58^{\prime} 28^{\prime} "-46^{\circ} 59^{\prime} 46^{\prime \prime} \mathrm{W}\right)$. Trata-se de uma área de 240 ha reflorestada em 2002 com alta diversidade de espécies nativas de floresta estacional semidecidual (Mandetta 2007, Barbosa et al. 2013, Comanetti et al. 2016).

O clima na região é o Cwa (inverno seco e verão úmido) segundo Köppen (1948), com temperatura média de $21^{\circ} \mathrm{C}$ e precipitação anual de $1.325 \mathrm{~mm}$ (EMBRAPA 2017).

Coleta e identificação - As coletas foram realizadas bimestralmente no período de um ano (outubro de 2015 a outubro de 2016), em dez (10) parcelas permanentes divididas em dois conjuntos amostrais (RPPN-A e RPPN-B), com duas (2) transecções (4 x $50 \mathrm{~m}$ ) inseridas em cada parcela, sendo a comunidade de Agaricomycetes da Reserva caracterizada de acordo com riqueza, abundância e grupos funcionais (Alcantara 2017). A avaliação do estado de preservação da área estudada foi realizada por meio da comparação dos resultados com aqueles obtidos por Abrahão et

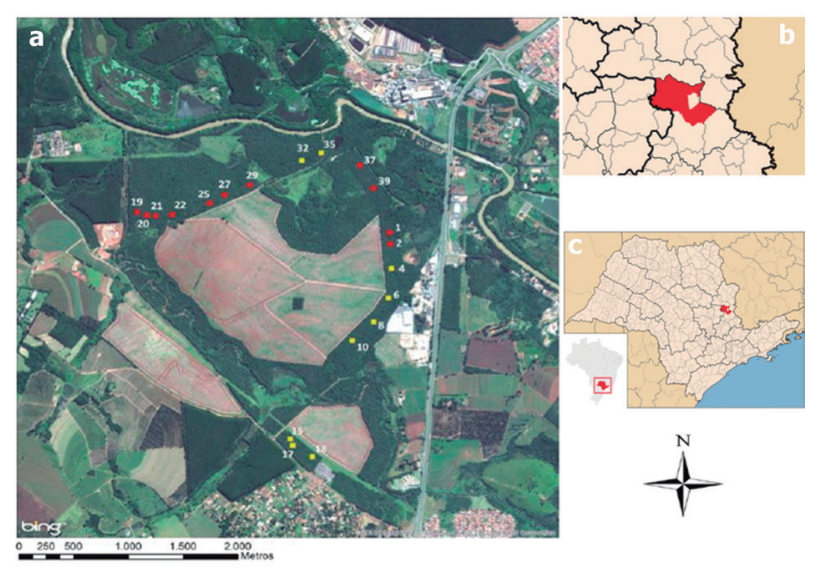

Figura 1. O local do estudo, Reserva Particular do Patrimônio Natural (RPPN) Parque Florestal São Marcelo. a. Localizado no município de Mogi-Guaçu. b. Estado de São Paulo, Brasil. c. (Barbosa et al. 2013).

Figure 1. The study site, Reserva Particular de Patrimônio Natural (RPPN) Parque Florestal São Marcelo. a. Located in the municipality of Mogi-Guaçu. b. São Paulo State, Brazil. c. (Barbosa et al. 2013). 
al. (2019), que realizou uma análise da ocorrência e distribuição de Agaricomycetes lignícolas, em uma área considerada preservada, de mesma formação vegetal no município de Mogi-Guaçu, a Reserva Biológica de Mogi-Guaçu.

A coleta e a herborização do material foram realizadas seguindo as técnicas recomendadas por Fidalgo \& Bononi (1984). A identificação dos espécimes foi baseada em caracteres macro e microscópicos dos basidiomas de acordo com Ryvarden (1991) e Teixeira (1995), como cor, tamanho, forma do píleo, forma e tamanho dos poros, em microscópio estéreoscópico Leica S6D. Para as análises microscópicas, secções transversais do basidioma foram preparadas em lâminas com solução de KOH a 5\% (Teixeira 1995). Reações amiloide e dextrinoide foram testadas no reagente de Melzer seguindo Gilbertson \& Ryvarden (1986). Observações e medições de estruturas microscópicas foram realizadas utilizando um microscópio óptico Leica DM 1000.

\section{Resultados e Discussão}

Durante o estudo sobre a compreensão da dinâmica da comunidade de macrofungos lignícolas e estabelecimento de parâmetros de monitoramento da Reserva Particular do Patrimônio Natural (RPPN) Parque Florestal São Marcelo (Alcantara 2017), um novo registro de Neofavolus subpurpurascens foi encontrado para o Estado de São Paulo.

Neste levantamento, um total de 224 espécimes de Agaricomycetes lignícolas (Basidiomycota) foi identificado em 41 espécies pertencentes a dez famílias e cinco ordens (Agaricales, Auriculariales, Gloeophyllales, Hymenochaetales e Polyporales), dos quais 11 foram de $N$. subpurpurascens (Alcantara 2017).

Neofavolus subpurpurascens (Murrill) Palacio \& Robledo in Phytotaxa. 405(4): 184. 2019.

$\equiv$ Polyporus subpurpurascens (Murrill) Ryvarden, Mycotaxon 23: 181, 1985.

三Favolus subpurpurascens (Murrill) Sacc. \& Trotter, Sylloge Fungorum 21: 357. 1912.

Tipo: JAMAICA, s.d., FS. Earle s.n. (tipo NY).

Figuras 2-3

Descrição e ilustração: Palacio et al. (2019), Coelho \& Silveira (2014).

Habitat: galho em decomposição; causador de podridão branca.
Material examinado selecionado: BRASIL. SÃo Paulo: Mogi-Guaçu, Parque Florestal São Marcelo, A.A. Alcantara, 26.X.2016, AL213, AL226, AL227 (SP).

Distribuição: Jamaica (2004, localidade tipo), América do Sul. No Brasil foi citada pela primeira vez como Polyporus subpurpurascens (Murrill) Ryvarden por Coelho \& Silveira (2014) para o Estado do Rio Grande do Sul.

Neofavolus subpurpurascens pode ser facilmente reconhecido em campo pelo basidioma frágil, estipitado, de coloração vinho a vermelho-escuro, himenóforo com poros favoides muito largos (Figuras 2-3). Coelho \& Silveira (2014, como $P$. subpurpurascens) descrevem essa espécie pela primeira vez no Brasil, no Estado do Rio Grande do Sul e apresentam ilustrações e descrição das principais características como: poros grandes, radialmente alongados $(1 / \mathrm{mm})$ ou maiores; basidiósporos subcilíndricos ca. 9,2 x 11,8 por 3,2 × $4 \mathrm{~mm}$. De acordo com Trierveiler-Pereira (2019, citado como Favolus subpurpurascens) trata-se de espécie comestível, apesar de não cultivada comercialmente.

O gênero Neofavolus foi proposto por Sotome et al. (2013) com base em resultados dos estudos detalhados das principais características taxonômicas e o auxílio da biologia molecular. Recentemente, estudos moleculares revelaram que Polyporus subpurpurascens (Murrill) Ryvarden faz parte do gênero Neofavolus, resultando em uma nova combinação (Palacio et al. 2019).

De acordo com Palacio et al. (2019), a espécie é rara com poucos registros além da localidade tipo (Jamanica), tendo sido registrada na Argentina, Bolívia e Brasil. No Estado de São Paulo há um relato de sua ocorrência em Angatuba em uma publicação sobre espécies comestíveis do município (TrierveilerPereira 2019). Apesar de apresentar ilustrações macroscópicas do basidioma e uma descrição sucinta da espécie, este trabalho não relaciona vouchers ou material examinado. A região de Angatuba está localizada na região sudoeste do Estado de São Paulo, cujas fisionomias principais são representadas pela Floresta Estacional Semidecidual e pelo Cerrado (Monteiro et al. 2009). Diversos levantamentos foram realizados em áreas com essa cobertura vegetal no Estado, mas nenhum relatou a ocorrência de Neofavolus subpurpurascens (Abrahão et al. 2009, 2012, 2019, Gugliotta et al. 2012, Maia et al. 2015). Assim, o presente trabalho considera esta a primeira 


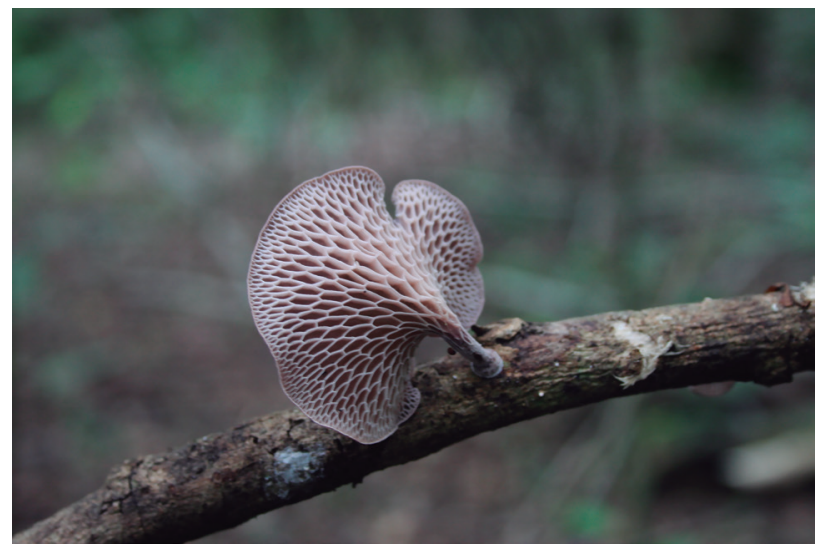

Figura 2. Neofavolus subpurpurascens, himenóforo poroide (al226).

Figure 2 Neofavolus subpurpurascens, poroid hymenophore (al226).

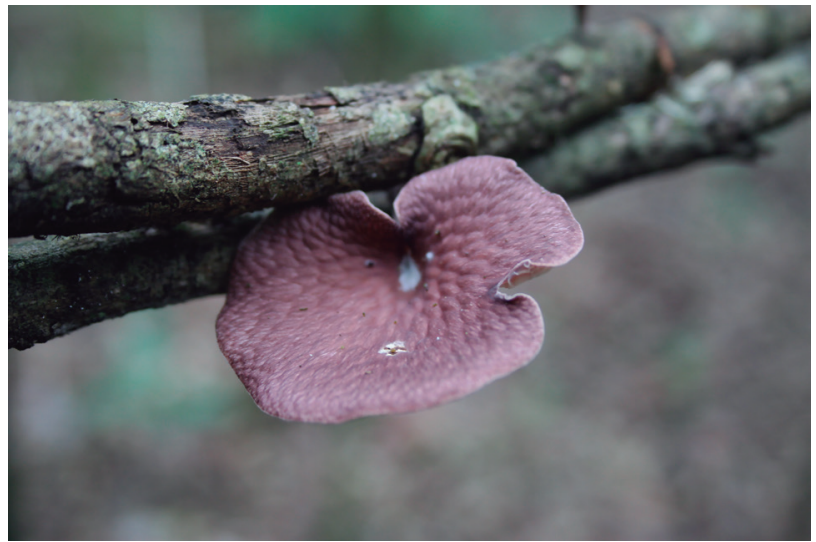

Figura 3. Neofavolus subpurpurascens, superfície do píleo (a1226).

Figure 3. Neofavolus subpurpurascens, pilear surface (a1226).

ocorrência comprovada da espécie no Estado de São Paulo e amplia a distribuição da mesma no Brasil, a qual estava restrita até o momento a dois espécimes coletados no Rio Grande do Sul (Coelho \& Silveira 2014, Palacio et al. 2019).

A presença desta e demais espécies de Agaricomycetes encontradas mostram o estabelecimento da comunidade fúngica na área restaurada e que a recuperação de áreas degradadas contribui para a manutenção da diversidade fúngica do Estado.

\section{Agradecimentos}

Agradecemos à International Paper do Brasil, pelo suporte, em especial ao Miguel Magela e João Machado. Aos funcionários do CERAD/IBt, pela ajuda e realização do projeto de pesquisa, em especial à Marcia Regina Ângelo e Fernando Cirilo. Aos colegas de laboratório Viviana Motato-Vásquez e Ricardo Matheus Pires, pelo auxílio na taxonomia. Ao CNPq e ao Programa de Pós-Graduação em Biodiversidade Vegetal e Meio Ambiente do Instituto de Botânica, pela bolsa de Mestrado concedida ao segundo Autor.

\section{Literatura citada}

Abrahão, M.C., Gugliotta, A.M. \& Gomes, E. 2009. Poliporóides (Basidiomycota) em fragmentos de mata no perímetro urbano de São José do Rio Preto, São Paulo, Brasil. Revista Brasileira de Botânica 32: 427-440.

Abrahão, M.C., Gugliotta, A.M. \& Bononi, V.L.R. 2012. Xylophilous Agaricomycetes (Basidiomycota) of the Brazilian Cerrado. Check List 8: 1102-1116.

Abrahão, M.C., Pires, R.M., Gugliotta, A.M., Gomes, E.P.C. \& Bononi, V.L.R. 2019. Wood-decay fungi (Agaricomycetes, Basidiomycota) in three physiognomies in the savannah region in Brazil. Hoehnea 46: e692018.

Alcantara, A.A. 2017. Diversidade de políporos (Basidiomycota) em uma área de reflorestamento com espécies nativas no município de Mogi-Guaçu, SP, Brasil. Dissertação de Mestrado, Instituto de Botânica, São Paulo.

Barbosa, L.M., Colmanetti, M.A.A., Shirasuna, R.T., Ortiz, P.R.T., Barbosa, T.C., Parajara, F.C., Barbosa, K.C., Casagrande, J.C., Couto, H.T.Z., Magela, M. \& Machado, J. 2013. Dez anos de pesquisa do Instituto de Botânica visando à restauração ecológica em áreas da International Paper do Brasil, em Mogi-Guaçu/SP. In: L.M. Barbosa (coord.). Políticas públicas para a restauração ecológica e conservação da biodiversidade. Instituto de Botânica/SMA, São Paulo, pp. 252-261.

Boddy, L., Frankland, J.C. \& van West, P. 2008. Ecology of Saprotrophic Basidiomycetes. Academic Press, London.

Bononi, V.L.R. 1984. Basidiomicetos do cerrado da Reserva Biológica de Moji-Guaçu, SP. Rickia 11: 1-25.

Coelho, G. \& Silveira, R.M.B. 2014. Taxonomy of Polyporus sensu lato and related genera (Basidiomycota) from Santa Maria, Southern Brazil. Ciência e Natura 36: 688-710.

Colmanetti, M.A.A. \& Barbosa, L.M. 2013. Fitossociologia e estrutura do estrato arbóreo de um reflorestamento com espécies nativas em Mogi-Guaçu, SP, Brasil. Hoehnea 40: 419-435.

Colmanetti, M.A., Barbosa, L.M., Shirasuna, R.T. \& Couto, H.T.Z. 2016. Phytosociology and structural characterization of woody regeneration from a reforestation with native species in southeastern Brazil. Revista Árvore 40: 209-218. 
Embrapa 2017. Banco de dados climáticos do Brasil. Disponível em http://www.cnpm.embrapa.br/projetos/ bdclima/balanco/resultados/sp/386/balanco.html (acesso em 08-VIII-2017).

Fidalgo, O. \& Bononi, V.L.R. 1984. Técnicas de coleta, preservação e herborização de material botânico. Manual n.4. Instituto de Botânica, São Paulo.

Gandolfi, S. 2013. Reflexões sobre as ações de restauração e definição de parâmetros de avaliação e monitoramento. In: L.M. Barbosa (coord.). Políticas públicas para a restauração ecológica e conservação da biodiversidade. Instituto de Botânica/SMA, São Paulo.

Gilbertson, R.L. \& Ryvarden, L. 1986. North American Polypores. 1 ed. Fungiflora, Oslo.

Gugliotta, A.M. \& Bononi, V.L.R. 1999. Polyporaceae do Parque Estadual da Ilha do Cardoso, São Paulo, Brasil. Boletim do Instituto de Botânica 12: 1-112.

Gugliotta, A.M., Poscolere, G.D. \& Campacci, T.V.S. 2011. Criptógamos do Parque Estadual das Fontes do Ipiranga, São Paulo, SP, Brasil. Fungos, 10: Ganodermataceae. Hoehnea 38: 687-695.

Gugliotta, A.M., Abrahão, M.C. \& Capelari, M. 2012. Basidiomicetos em fragmentos florestais remanescentes da região noroeste do estado de São Paulo. In: O. Necchi Jr. (ed.). Fauna e Flora de Fragmentos Florestais Remanescentes da Região Noroeste do Estado de São Paulo. Holos Editora, Ribeirão Preto, pp. 93-103.

Köppen, W. 1948. Climatologia: con un estudio de los climas de la tierra. Fondo de Cultura Econômica, México.

Leal, G.R. \& Gugliotta, A.M. 2008. Criptógamos do Parque Estadual das Fontes do Ipiranga, São Paulo, SP. Fungos, 9: Meripilaceae. Hoehnea 35: 99-110.

Maia, L.C., Carvalho Jr., A.A., Cavalcanti, L.D.H., Gugliotta, A.M., Drechsler-Santos, E.R., Santiago, A.L.M.A., Giachini, A.J., Soares, A.M.S., GomesSilva, A.C., Magnago, A.C., Goto, B.T., Lira, C.R.S., Salvador-Montoya, C.A., Pires-Zottarelli, C.L.A., Silva, D.K.A., Soares, D.J., Rezende, D.H.C., Luz, E.D.M.N., Gumboski, E.L., Wartchow, F., Karstedt, F., Freire, F.M., Coutinho, F.P., Melo, G.S.N., Sotão, H.M.P., Baseia, I.G., Pereira, J., Oliveira, J.J.S., Souza, J.F., Bezerra, J.L., Araujo Neta, L.S., Pfenning, L.H., Gusmão, L.F.P., Neves, M.A., Capelari, M., Jaeger, M.C.W., Pulgarín, M.P., Menolli Jr., N., Medeiros, P.S., Friedrich, R.C.S., Chikowski, R.S., Pires, R.M., Melo, R.F., Silveira, R.M.B., Urrea-Valencia, S.,; Cortez, V.G. \& Silva, V.F. 2015. Diversity of Brazilian Fungi. Rodriguésia 66: 1033-1045.
Mandetta, E.C.N. 2007. Avaliação florística e de aspectos da estrutura da comunidade de um reflorestamento com dois anos e meio de implantação no município de MogiGuaçu/SP. Dissertação de Mestrado, Universidade Estadual Paulista, Rio Claro.

Monteiro, C.H.B., Prado, B.H.S. \& Dias, A.C. 2009. Plano de Manejo Estação Ecológica de Angatuba. Instituto Florestal, São Paulo.

Motato-Vásquez, V. \& Gugliotta, A.M. 2013. Polypores from an Atlantic rainforest area in southeast Brazil: resupinate species. Revista Brasileira de Botânica 36: 211-221.

Motato-Vásquez, V., Pires, R.M. \& Gugliotta, A.M. 2014. Polypores from an Atlantic rainforest area in southeast Brazil: pileate species. Revista Brasileira de Botânica 38: 149-164.

Palacio, M., Silveira, R.M.B. \& Robledo, G.L. 2019. Neofavolus subpurpurascens comb. nov., with new records from the Neotropics. Phytotaxa 405: 180-186.

Pires, R.M. \& Gugliotta, A.M. 2016. Poroid Hymenochaetaceae (Basidiomycota) from Parque Estadual da Serra do Mar, Núcleo Santa Virgínia, São Paulo, Brazil. Rodriguésia 63: 667-676.

Rayner, A.D.M. 1995. Fungi, a vital component of ecosystem function in woodland. In: D. Allsopp, R.R. Colwell, D.L. Hawksworth (ed.). Microbial Diversity and Ecosystem Function. University Press, Cambridge, pp. 231-251.

Ryvarden, L. 1985. Type studies in the Polyporaceae 17. Species described by W.A. Murrill. Mycotaxon 23: $169-198$

Ryvarden, L. 1991. Genera of Polypores. Nomenclature and Taxonomy. Synopsis Fungorum 5: 1-363.

Ryvarden, L. 2004. Neotropical Polypores. Part 1. Introduction, Ganodermataceae \& Hymenochaetaceae. Synopsis Fungorum 19: 1-227.

Silveira, M., Trevelin, L., Port-Carvalho, M., Godoi, S., Mandetta, E.M. \& Cruz-Neto, A.P. 2011. Frugivory by phyllostomid bats (Mammalia: Chiroptera) in a restored area in Southeast Brazil. Acta Oecologica 37: 31-36.

Sotome, K., Akagi, Y., Lee, S.S., Ishikawa, N.K. \& Hattori, T. 2013. Taxonomic study of Favolus and Neofavolus gen. nov. segregated from Polyporus (Basidiomycota, Polyporales). Fungal Diversity 58: 245-266.

Teixeira, A.R. 1995. Método para estudo das hifas do basidiocarpo de fungos poliporáceos. Manual n.6. Instituto de Botânica, São Paulo.

Trierveiler-Pereira L. 2019. FANCs de Angatuba: Fungos Alimentícios Não Convencionais de Angatuba e região. PLUS/Simplíssimo, Porto Alegre. 